\title{
Ab-initio Calculations of Diffusion Lengths and Diffusivities for Singlet Excitons in Organic Materials
}

Fernando Teixeira Bueno* (IC) ${ }^{1}$, Leonardo Evaristo Sousa $(\mathrm{PG})^{1}$, Demétrio Antônio da Silva Filho (PQ) ${ }^{1}$, Pedro Henrique de Oliveira Neto (PQ) ${ }^{1}$

f.bueno1998@gmail.com

${ }^{1}$ Physics Institute, University of Brasília;

Keywords: Exciton diffusion, diffusion length, organic materials

\section{Introduction}

Exciton diffusion plays a major role in the working of organic optoelectronic devices. For singlet excitons, the mechanism behind diffusion is the Förster resonance energy transfer (FRET). In FRET, excitation energy is transferred non-radiatively from donor to acceptor molecule as long as there is overlap between the emission and absorption spectra of both species. This overlap, along with intermolecular distances and relative orientation between molecular dipoles are determinant to the efficiency of the process, which, in its turn, determines exciton diffusion length. In this work, a combination of quantum chemistry calculations and analytical random walk model is employed to provide ab initio estimations of singlet exciton diffusion constants and diffusion lengths for several organic molecules of interest to the field of organic electronics. The protocol developed here is general and may be used as a tool to assess, before synthesis, the suitability of molecules to applications for which large diffusion lengths are required.

\section{Methodology}

Density Functional Theory (DFT) and Time-Dependent Functional Theory (TD-DFT) are used to perform geometry optimizations of all molecules in the ground state and first excited state, respectively. Frequency calculations are then employed to check whether the optimized geometries correspond to minima of the potential energy surfaces. B3LYP, CAM-B3LYP, M062X and WB97XD functionals are used depending on the characteristics of the molecules. The 6-31G(d,p) basis set is used throughout. All DFT and TD-DFT calculations are performed using the Gaussian09 program suite. Once optimizations and normal modes have been obtained, absorption and emission spectra are simulated using the nuclear ensemble method as implemented in the Newton-X software. These spectra are used to calculate the Förster radius for each material in conjunction with information on the molecular arrangements typical to each molecule, be it amorphous or crystalline. Finally, results are plugged into the analytical random walk model to yield diffusion coefficients and diffusion lengths.

\section{Results}

Figure 1 shows the absorption and emission spectra for the para-hexaphenyl (P6P) molecule. The overlap shows that Förster transfer between P6P molecules is possible. 
Figure 1

From

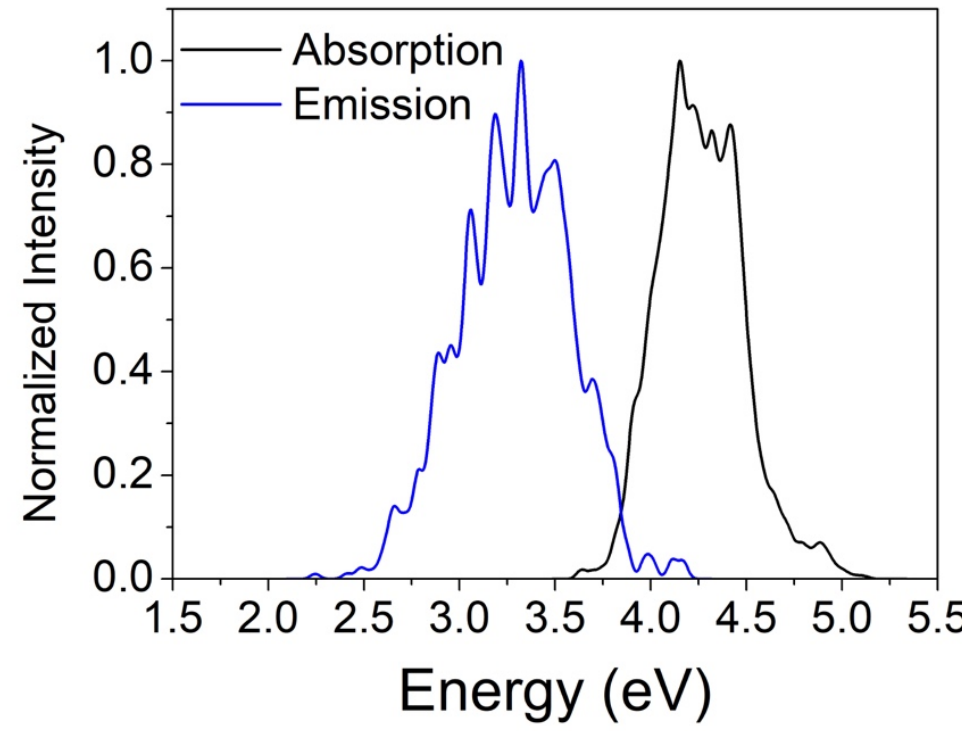

these the

Förster radius for this transition is evaluated. Using this parameter, the corresponding diffusion length and diffusion constant were evaluated by employing the properties of the Wiener process.

\section{Discussion}

Results show diffusion lengths within typical values observed for organic materials. This goes to show that the method employed in this study may indeed be useful to predict exciton diffusion behavior in organic materials before synthesis.

\section{Conclusions}

In this work, we demonstrated an ab initio method for calculating the diffusion properties of singlet excitons in organic materials. By simulating absorption and emission spectra, Förster radii for a large set of molecules were calculated. These, along with analytical considerations allowed us to obtain estimates of diffusion lengths and diffusion constants in agreement with experimental results.

\section{Acknowledgments}

The authors gratefully acknowledge the financial support from CAPES and FAP-DF.

\section{References}

EVARISTO DE SOUSA, LEONARDO ; Ferreira da Cunha, Wiliam ; ANTÔNIO DA SILVA FILHO, DEMÉTRIO ; DE OLIVEIRA NETO, PEDRO HENRIQUE . Biexciton cascade emission in multilayered organic nanofibers. APPLIED PHYSICS LETTERS JCR, v. 112, p. 143301, 2018.

EVARISTO DE SOUSA, LEONARDO; DE OLIVEIRA NETO, PEDRO HENRIQUE ; KJELSTRUP-HANSEN, JAKOB ; ANTÔNIO DA SILVA FILHO, DEMÉTRIO . 
VII SeedMol, 17 a 21 de setembro de 2018

DOI 10.21826/97885638003740]

Modeling temperature dependent singlet exciton dynamics in multilayered organic nanofibers. JOURNAL OF CHEMICAL PHYSICS JCR, v. 148, p. 204101, 2018. 